\title{
Molecular dynamics studies of the melting of butane and hexane monolayers adsorbed on the basal-plane surface of graphite
}

\author{
Flemming Y. Hansen \\ Fysisk-Kemisk Institut, The Technical University of Denmark, Denmarks Tekniske Hbjskole 206, DK- \\ 2800 Lyngby, Denmark \\ J. C. Newton ${ }^{\text {a) }}$ and H. Taub \\ Department of Physics and Astronomy, University of Missouri-Columbia, Columbia, Missouri 65211
}

(Received 8 September 1992; accepted 27 November 1992)

\begin{abstract}
The effect of molecular steric properties on the melting of quasi-two-dimensional solids is investigated by comparing results of molecular dynamics simulations of the melting of butane and hexane monolayers adsorbed on the basal-plane surface of graphite. These molecules differ only in their length, being members of the $n$-alkane series $\left[\mathrm{CH}_{3}\left(\mathrm{CH}_{2}\right)_{n-2} \mathrm{CH}_{3}\right]$ where $n=4$ for butane and $n=6$ for hexane. The simulations employ a skeletal model, which does not include the hydrogen atoms explicitly, to represent the intermolecular and molecule-substrate interactions. Nearest-neighbor intramolecular bonds are fixed in length, but the molecular flexibility is preserved by allowing the bend and dihedral torsion angles to vary. The simulations show a qualitatively different melting behavior for the butane and hexane monolayers consistent with neutron and x-ray scattering experiments. The melting of the low-temperature herringbone ( $\mathrm{HB}$ ) phase of the butane monolayer is abrupt and characterized by a simultaneous breakdown of translational order and the orientational order of the molecules about the surface normal. In contrast, the hexane monolayer exhibits polymorphism in that the solid $\mathrm{HB}$ phase transforms to a rectangular-centered structure with a short coherence length in coexistence with a fluid phase. A significant result of the simulations is that they demonstrate the importance of molecular flexibility on the nature of the melting transition. The formation of gauche molecules is essential for the melting process in the hexane monolayer but unimportant for butane. The effect of molecular length on the qualitative nature of the melting process is discussed for both monolayers.
\end{abstract}

\section{INTRODUCTION}

For some time we have been interested in the question of how the melting of quasi-two-dimensional (2D) physisorbed monolayers is influenced by the steric properties of the constituent molecules. ${ }^{1}$ It seems plausible that the melting behavior of 2D solids should depend more strongly on molecular shape than in the bulk phase. For example, if one considers a rigid rod-shaped molecule adsorbed with its long axis parallel to a surface, the potential energy barrier to rotation about the surface normal could be quite large compared to the steric hindrance encountered in the bulk phase. This is because molecules in the bulk do not remain confined to or near a single plane. In general, orientational and translational disorder can be achieved more easily than in a monolayer due to exchange of molecules between neighboring layers.

Our approach to studying the effect of molecular steric properties on monolayer melting has been to consider a series of isostructural rod-shaped molecules, the $n$-alkanes $\left[\mathrm{CH}_{3}\left(\mathrm{CH}_{2}\right)_{n-2} \mathrm{CH}_{3}\right]$, physisorbed on a graphite basalplane surface. We have investigated the dependence of their monolayer melting behavior on the length of the molecule $(n)$ using both neutron scattering and x-ray diffraction techniques. ${ }^{1}$ The experiments show a monotonic in-

\footnotetext{
a) Present address: Campus Computing, University of Missouri-Columbia, Columbia, MO 65211.
}

crease in the monolayer melting point as the length of the molecule increases in the series ethane $(n=2)$, butane ( $n$ $=4)$, and hexane $(n=6)$. For hexane, the ratio of the monolayer-to-bulk melting point approaches unity compared to values of 0.5 to 0.6 typical of nearly spherical molecules. The lower melting point ratio for spherical molecules can be explained qualitatively by the fewer number of nearest neighbors in 2D compared to 3D. With rodshaped molecules, this effect is presumably opposed by increased steric hindrance to in-plane orientational disorder.

In addition to their different melting points, the butane and hexane monolayers appear to differ in the qualitative nature of the melting process and their structure above the melting transition. Neutron and $\mathrm{x}$-ray diffraction experiments have shown both butane $e^{2,3}$ and hexane ${ }^{3-5}$ to have rectangular-commensurate orientationally ordered structures in which the molecules lie with their long axis parallel to the surface in a herringbone arrangement. ${ }^{1}$ Yet, the butane monolayer has an abrupt first-order melting, ${ }^{1,6,7}$ whereas that of hexane appears to be more gradual with the monolayer exhibiting an unusual degree of translational order at high temperatures. ${ }^{3,5}$ From the neutron and $\mathrm{x}$-ray diffraction patterns, it proved difficult to arrive at a unique interpretation of the high-temperature hexane monolayer diffraction patterns.

Another case where steric effects are believed responsible for qualitatively different melting behavior is that of 
methane and ethylene physisorbed on graphite. The methane monolayer clearly has a first-order melting transition, ${ }^{8}$ whereas that of ethylene appears continuous., ${ }^{9,10}$ It was suggested that different mechanisms of vacancy formation are involved in the monolayer melting of the planar ethylene molecule compared to nearly spherical methane. ${ }^{10}$

The molecular dynamics simulations presented here were strongly motivated by the previous diffraction work with butane and hexane monolayers on graphite. ${ }^{1-7}$ In particular, we were interested in the case of the longer hexane molecule for which steric effects on the monolayer melting should be most pronounced. The butane monolayer provides an interesting comparison especially since the diffraction experiments had indicated a qualitatively different melting behavior. We felt that a monolayer of the still shorter ethane molecule would actually be more difficult to treat initially since it could form structures with its long axis either parallel or perpendicular to the surface. ${ }^{111}$ Also, the corrugation of the ethane-graphite potential, which is not known well, seemed important to understanding the so-called "lattice fluid" phase observed at high temperatures. ${ }^{12,13}$

The specific objectives of this study were to address the following questions: (1) Why do the butane and hexane monolayers behave differently upon heating? (2) What is the structure of the hexane monolayer above $170 \mathrm{~K}$ where a qualitative change in the diffraction patterns occurs? (3) Do the butane and hexane molecules become rotationally disordered about their long axis while still in the lowtemperature solid monolayer phase? (4) Is the flexibility of the molecules important in understanding the melting process? The last question is of special interest since it is difficult to extract such information from elastic diffraction experiments.

The work presented here is the first molecular dynamics simulation of the melting transition in physisorbed monolayers of rod-shaped hydrocarbon molecules. Moller and Klein ${ }^{14}$ have investigated the structure and dynamics of the three low-temperature monolayer phases of ethane on graphite ( $S 1, S 2$, and $S 3$ ), but not melting. Also, Leggetter and Tildesley ${ }^{15}$ have simulated fluid monolayers of butane and decane at room temperature but not the lowtemperature solid phases.

Briefer accounts of some of this work have appeared in Refs. 16 and 17. Our purpose here is to give a more complete description of the methods used in the molecular dynamics simulations. Our presentation is organized as follows: In the next section, we discuss the skeletal model of the alkane molecules used to represent the intermolecular and molecule-substrate interactions. We also describe the intramolecular potentials used for the dihedral torsions and angle bends. Section III gives a brief account of the simulation procedure. The constraint dynamics used is described along with the choice of the simulation box size and the method used to generate the starting configuration. The data obtained from the simulations are presented in Sec. IV and the results discussed in Sec. V. Finally, Sec. VI contains a summary, conclusions, and a description of future studies planned.
TABLE I. Comparison of the principal moments of inertia $I_{j}(j=1,2,3$; $I_{1}<I_{2}<I_{3}$ ) calculated from the skeletal model with the actual moments for three different alkane molecules.

\begin{tabular}{lcc}
\hline \hline Molecule & $j$ & $I_{j}$ (skeletal) $/ I_{j}$ (actual) \\
\hline Butane & 1 & 0.39 \\
& 2 & 0.88 \\
& 3 & 0.88 \\
Hexane & 1 & 0.42 \\
& 2 & 0.93 \\
Decane & 3 & 0.93 \\
& 1 & 0.43 \\
& 2 & 0.96 \\
\hline \hline
\end{tabular}

\section{REPRESENTATION OF THE INTERACTIONS}

The skeletal model of alkane molecules has been successful in simulating the collective behavior of bulk hydrocarbons. ${ }^{18-20}$ In the model, the methyl and methylene groups are replaced by single force centers or pseudoatoms located at the carbon atom positions. The mass assigned to the pseudoatoms is 15 and 14 amu for the methyl and methylene groups, respectively. The reasons for using the skeletal model in our simulation of the butane and hexane monolayers is much the same as for bulk systems: (1) reduction of the number of atoms in the film greatly shortens computation time; (2) the absence of hydrogen atoms in the molecules should not affect the calculated monolayer structure as long as the van der Waals dimensions of the molecules are reproduced well; and (3) the neglect of the fast intramolecular $\mathrm{C}-\mathrm{H}$ stretching modes does not affect the modeling of the dynamical properties of the films near melting.

Although it gives the correct mass distribution along the chain, we would like to point out that the skeletal model gives a poor representation of the alkane molecules' moments of inertia. Table I compares the true principal moments with those of the skeletal model for butane, hexane, and decane. The largest discrepancy occurs for the smallest moment of inertia which is about the long axis of the molecule and for which the model gives a value about a factor of 2.5 too small. Such errors must be considered in the calculation of rotational motion; however, it is unimportant for calculating equilibrium properties of a classical system which is our focus here. This is because the moments of inertia appear in the standard chemical potential which is the same for all phases and hence of no importance in the analysis of phase equilibria. ${ }^{21}$

In implementing the skeletal model, we fix the length of the nearest-neighbor bonds while allowing the bend and dihedral torsion angles to vary. Previous simulations of bulk alkane phases ${ }^{22}$ have shown that a fixed bond length does not alter the results significantly while allowing larger time steps to be used in the integration of the equations of motion. They also showed that the molecular flexibility manifest in angle-bending and torsional motion was essen: tial for a realistic simulation of the system. 
TABLE II. Comparison of experimental (expt.) structural parameters with those calculated (calc.) from the skeletal model of the butane and hexane monolayers at low temperature. The center-of-mass coordinates of the molecules denoted by $(x, y)$ are in $\AA$, and the angular parameters (defined in Fig. 3 ) are in degrees. The lattice constants $a$ and $b(\AA)$ are defined in Fig. 5. Experimental values are from Ref. 3 .

\begin{tabular}{|c|c|c|c|c|c|c|c|}
\hline \multirow{2}{*}{ Monolayer } & \multirow[b]{2}{*}{ Parameter } & \multicolumn{2}{|c|}{1} & \multicolumn{2}{|c|}{2} & \multicolumn{2}{|c|}{ Lattice } \\
\hline & & Calc. & Expt. & Calc. & Expt. & Calc. & Expt. \\
\hline \multirow[t]{7}{*}{ Butane } & $x$ & 0.0 & 0.0 & 3.89 & 3.80 & $\cdots$ & $\cdots$ \\
\hline & $y$ & 0.0 & 0.0 & 4.28 & 4.26 & $\cdots$ & $\cdots$ \\
\hline & $\psi$ & -90 & -90 & 90 & 90 & $\therefore$ & $\ldots$ \\
\hline & $\theta$ & 0.0 & 0.0 & 0.0 & 0.0 & - & $\ldots$ \\
\hline & $\phi$ & -22 & -12 & 22 & 12 & $\cdots$ & .. \\
\hline & $a$ & $\ldots$ & $\ldots$ & $\ldots$ & $\ldots$ & 7.88 & 7.60 \\
\hline & $b$ & $\cdots$ & $\cdots$ & $\cdots$ & $\cdots$ & 8.55 & 8.52 \\
\hline \multirow[t]{7}{*}{ Hexane } & $x$ & 0.0 & 0.0 & 8.53 & 8.52 & $\cdots$ & $\cdots$ \\
\hline & $y$ & 0.0 & 0.0 & 2.68 & 2.46 & $\cdots$ & $\cdots$ \\
\hline & $\psi$ & -90 & -90 & 90 & 90 & $\cdots$ & $\ldots$ \\
\hline & $\theta$ & 0.0 & 0.0 & 0.0 & 0.0 & $\ldots$ & $\cdots$ \\
\hline & $\phi$ & -65 & -67 & 65 & 67 & $\ldots$ & $\ldots$ \\
\hline & $a$ & $\ldots$ & $\ldots$ & $\cdots$ & $\ldots$ & 5.37 & 4.92 \\
\hline & $b$ & $\cdots$ & $\ldots \ldots$ & $\cdots$ & $\cdots$ & $\therefore-17.06$ & 17.04 \\
\hline
\end{tabular}

The intermolecular interactions are described as a sum of pair potentials $v_{i j}$ between pseudoatoms where no distinction is made between the methyl and methylene groups. The $v_{i j}$ used in this work are simple 6-12 LennardJones potentials,

$$
v_{i j}=4 \epsilon\left[\left(\frac{\sigma}{r_{i j}}\right)^{12}-\left(\frac{\sigma}{r_{i j}}\right)^{6}\right]
$$

with $\epsilon=72 \mathrm{~K}$ and $\sigma=3.92 \AA$, and where $r_{i j}$ is the distance between atoms $i$ and $j$ in two different molecules. This is the same potential used in the study of bulk hydrocarbon phases by Ryckaert and Bellemanns. ${ }^{23}$ We have used a cutoff distance of $2.5 \sigma$ beyond which the interaction between two atoms is neglected. At this distance, $v_{i j}$ is $1.6 \%$ of $\epsilon$.

The molecule-surface interaction is also described as a sum of 6-12 Lennard-Jones atom-atom potentials ${ }^{15}$ with $\epsilon_{s}=90 \mathrm{~K}$ and $\sigma_{s}=3.60 \AA$, where the values of these parameters are based on experimentally determined adsorption energies. Following Steele's method, ${ }^{24}$ the translational symmetry of the surface is used to convert the atomsurface energy from a summation over atom pairs to one over a relatively small number of reciprocal lattice vectors of the graphite surface. The number of these increases as a pseudoatom approaches the surface and is determined by a convergence criterion for the energy sum. The number of layers in the graphite crystal required for energy convergence also varies with the pseudoatom distance from the surface and is typically 7-12 layers. The atom-surface potential $u(z, \tau)$ is given by the expression

$$
\begin{aligned}
\frac{u(z, \tau)}{\epsilon_{s}}= & \frac{2 \pi}{A_{s}}\left[n\left(\frac{2}{5} \frac{\sigma_{s}^{12}}{z^{10}}-\frac{\sigma_{s}^{6}}{z^{4}}\right)+\sum_{g \neq 0} \sum_{i=1}^{n} \exp \left[i \mathrm{~g} \cdot\left(\tau-\tau_{\mathrm{i}}\right)\right]\right. \\
& \left.\cdot\left[\frac{\sigma_{s}}{30}\left(\frac{g}{2 z}\right)^{5} K_{5}(g z)-2 \sigma_{s}^{6}\left(\frac{g}{2 z}\right)^{2} K_{2}(g z)\right]\right],
\end{aligned}
$$

where $n(=2)$ is the number of carbon atoms in the unit cell of the graphite basal plane and $\tau_{\mathrm{i}}$ is the (2D) position vector of the $i$ th carbon atom in the surface unit cell of area $A_{s}$. The projection of the pseudoatom position vector on the surface is given by $\tau$ and $\mathbf{z}$ is its height above the surface. $g$ is a reciprocal lattice vector of the graphite surface and $K_{i}$ is the modified Bessel function of the second kind. ${ }^{25}$ In the lowest energy state, both the butane and hexane molecules are in the trans configuration with the plane of the pseudoatoms parallel to the surface at a height of $3.58 \AA$.

We have also tested the potentials in a static calculation of the zero-temperature structure of the butane and hexane monolayers performed with rigid molecules. Structural parameters calculated in the skeletal model for the low-temperature herringbone phases are compared in Table II with values obtained from neutron diffraction experiments. For both butane and hexane, the calculations predict the observed commensurability of the monolayers with the substrate in the graphite [100] direction ( $y$ axis in Fig. 5 ) to within the calculational uncertainty. However, in the graphite [110] direction ( $x$ axis in Fig. 5), the calculated lattice constant is larger than that observed (by $4 \%$ for butane and $9 \%$ for hexane). ${ }^{26} \mathrm{~A}$ similar discrepancy was found when the $\mathrm{H}$ atoms were explicitly included in the molecules, so we do not believe this to be a major deficiency of the skeletal model. The molecular orientations inferred from profile analysis of the neutron diffraction patterns are in very close agreement with those calculatedthe only exception being the in-plane azimuthal orientation of the butane molecule where there is a discrepancy of $10^{\circ}$.

The two intramolecular potentials of importance in the simulations are those corresponding to the angle-bend and dihedral torsional motion of the pseudoatoms. The anglebend potential is that used by Weber. ${ }^{19}$ It is quadratic in the cosine of the bend angle $\theta_{i}^{\prime}$, 


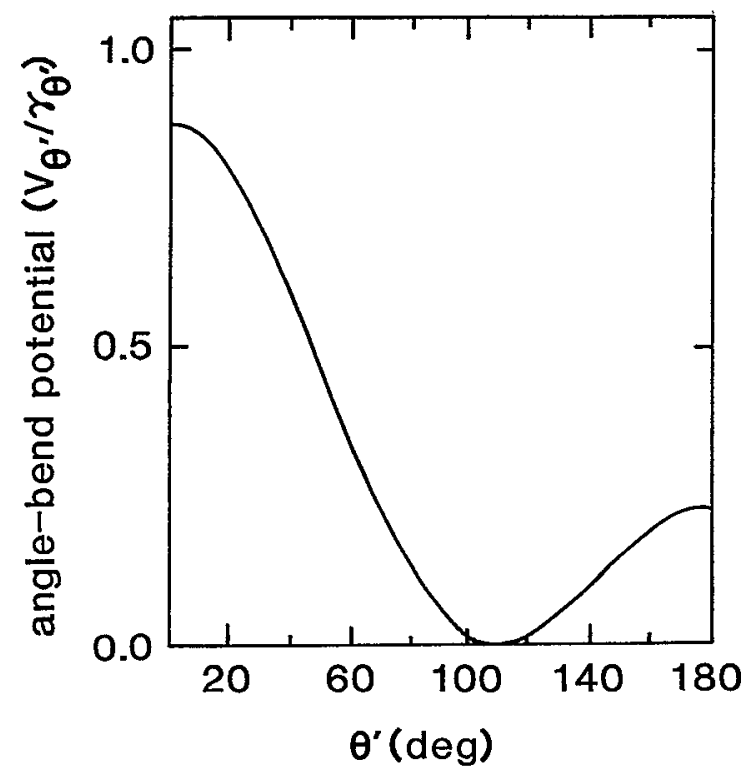

FIG. 1. Bend-angle potential $V_{\theta^{\prime}} / \gamma_{\theta^{\prime}}$ defined in Eq. (3) where $\theta^{\prime}$ is the bend angle and $\gamma_{\theta^{\prime}}$ is the bend-angle force constant.

$$
V_{\theta^{\prime}}=0.5 \gamma_{\theta^{\prime}}\left(\cos \theta_{0}^{\prime}-\cos \theta_{i}^{\prime}\right)^{2},
$$

where $\gamma_{\theta^{\prime}}$ is the force constant and $\theta_{0}^{\prime}$ is the equilibrium bend angle. The bend angle can be computed from the relation

$$
\cos \theta_{i}^{\prime}=-\frac{\mathbf{b}_{i} \cdot \mathbf{b}_{i+1}}{\left|\mathbf{b}_{i}\right| \cdot\left|\mathbf{b}_{i+1}\right|},
$$

where $\mathbf{b}_{i}=\mathbf{r}_{i+1}-\mathbf{r}_{i}$ is the bond vector between pseudoatoms at positions $\mathbf{r}_{i}$ and $\mathbf{r}_{i+1}$.

The angle-bend potential is plotted in Fig. 1 for an equilibrium bend angle $\theta_{0}^{\prime}=109^{\circ}$ and force constant $\gamma_{\theta^{\prime}}=1.56 \times 10^{4} \mathrm{~K}$. There are two maxima; however, the one at $\theta^{\prime}=0$ is unphysical since it corresponds to an overlap of the two atoms. This is of no importance for the simulation, since its energy is so high that $\theta^{\prime}$ values never come near this region. The other maximum at $180^{\circ}$ is the energy barrier to inversion of the bend angle.

The dihedral-torsion potential $V_{\text {dih }}$ associated with bond rotation is usually given in one of two forms:

$$
\begin{aligned}
& V_{\mathrm{dih}}\left(\phi^{\prime}\right)=\sum_{k=1}^{m} \frac{v_{k}}{2}\left[1-\cos \left(k \phi^{\prime}\right)\right], \\
& V_{\mathrm{dih}}\left(\phi^{\prime}\right)=\sum_{k=1}^{m} c_{k} \cos \left(k \phi^{\prime}\right),
\end{aligned}
$$

where $\phi^{\prime}$ is the dihedral-torsion angle and $m$ is of the order of $5-10 . .^{27,28}$ Remarkably, $V_{\text {dih }}$ is not known with great accuracy, although there have been several experimental and theoretical determinations. It is difficult to infer the overall shape of the potential experimentally from measurements of transitions between only a few vibrational levels of the molecule in the trans and gauche conformations. The range of calculated values has several origins: the use of different basis sets for the molecular wave func- tions and the differing treatment of or neglect of relaxation in the methyl and methylene groups and the bend angles.

With both angle-bend and dihedral-torsional motion present in our simulations, we include a coupling between these two variables in the expression for $V_{\text {din }}$ which does not appear in Eqs. (5) and (6). Following Weber, ${ }^{19}$ we write the dihedral potential as a sum of two terms,

$$
V_{\mathrm{dih}}=V_{\mathrm{tor}}+V_{\mathrm{LJ}}
$$

where $V_{\text {tor }}$ represents the energy cost to rotate about the covalent bond. It involves all four atoms necessary to specify a torsion angle and is given by

$$
V_{\text {tor }}=\gamma_{\phi^{\prime}}\left(1-\xi_{i}\right)\left(\xi_{i}^{2}+a_{1} \xi_{i}+a_{0}\right),
$$

where

$$
\begin{aligned}
\xi_{i} & =\frac{\mathbf{b}_{i} \cdot \mathbf{b}_{i+2}}{\left|\mathbf{b}_{i}\right| \cdot\left|\mathbf{b}_{i+2}\right|} \\
& =\frac{\mathbf{b}_{i} \cdot \mathbf{b}_{i+2}}{b^{2}} \\
& =\cos \theta_{i}^{\prime} \cos \theta_{i+1}^{\prime}-\sin \theta_{i}^{\prime} \sin \theta_{i+1}^{\prime} \cos \phi_{i}^{\prime},
\end{aligned}
$$

and $\mathbf{b}_{i}$ and $\theta_{i}^{\prime}$ are as previously defined in Eq. (4). Note that the bond lengths $b_{i}$ defining $\xi_{i}$ are fixed in length. For $\xi_{i}=1$ in Eq. (8), $V_{\text {tor }}=0$ corresponding to a dihedral angle $\phi_{i}^{\prime}=180^{\circ}$ when $\theta_{i}^{\prime}=\theta_{i+1}^{\prime}$. At this potential minimum the molecule is in the trans conformation. One can also show from Eq. (8) that the other minimum in the potential corresponds to the gauche conformation whose position $\left(\phi^{\prime} \approx \pm 60^{\circ}\right)$ is primarily determined by the $a_{1}$ parameter while the energy of the gauche state depends on both the $a_{0}$ and $a_{1}$ parameters. The barrier for the trans-gauche transition is controlled by $\gamma_{\phi^{\prime}}$.

The other part of the dihedral potential, $V_{\mathrm{LJ}}$, is an atom-atom potential acting between force centers separated by two or more pseudoatoms along the chain. For example, in butane it describes the interaction between the pseudoatoms at each end of the molecule. Since $V_{\mathrm{LJ}}$ depends on the separation of the two pseudoatoms, it will also depend on the bend angles. We choose $V_{\mathrm{LJ}}$ as a simple Lennard-Jones potential

$$
V_{\mathrm{LJ}}=4 E_{\mathrm{LJ}}\left[\left(\frac{\sigma_{\mathrm{LJ}}}{r}\right)^{12}-\left(\frac{\sigma_{\mathrm{LJ}}}{r}\right)^{6}\right] .
$$

The $V_{\mathrm{LJ}}$ term in the potential Eq. (7) primarily determines the $c i s$ barrier at $\phi^{\prime}=0$. The repulsive part of $V_{\mathrm{LJ}}$ and hence the barrier can increase greatly as the $\theta^{\prime}$ (bend) angles decrease, reducing the separation between the end members of a group of four pseudoatoms defining a torsional angle. This effect also shifts the position of the gauche state to slightly higher $\phi^{\prime}$ angles and can alter its energy.

The potentials used in this simulation are illustrated in Fig. 2(a). The dashed curve is $V_{\text {tor }}$ and the solid curve $V_{\text {dih }}$. It can be seen that at $\phi^{\prime}$ angles above $\sim 100^{\circ}$ the $V_{L J}$ term is negligible. The parametrization of the potential is based on the most recent experimental ${ }^{29}$ and theoretical ${ }^{30}$ investigations. We have used 

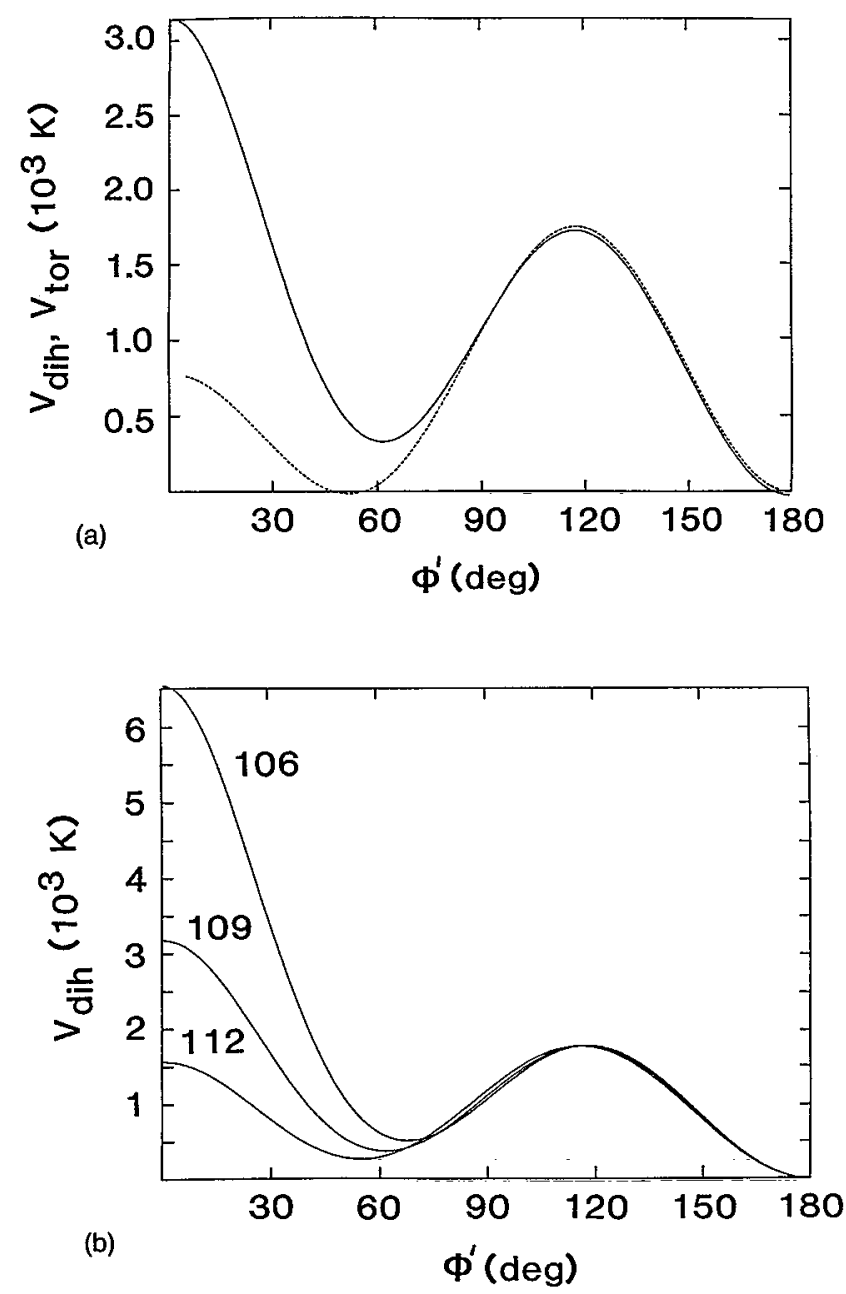

FIG. 2. (a) The dihedral-torsion potential $V_{\text {dih }}$ as a function of the dihedral-torsion angle $\phi^{\prime}$. The dashed curve is $V_{\text {tor }}$ defined in Eqs. (7) and (8). (b) Dependence of $V_{\text {dih }}$ on the bend angles. The numbers on the curves indicate the magnitude of the bend angles (in degrees) assumed to be the same within a molecule. In both (a) and (b), the potential energy function is expressed in units of degrees Kelvin. The peaks at $\phi^{\prime}=0$ and $\phi^{\prime}=120^{\circ}$ represent the gauche-gauche and trans-gauche barriers, respectively.

$$
\begin{aligned}
& \gamma_{\phi^{\prime}}=3900 \mathrm{~K}, \\
& a_{1}=0.9, \\
& a_{0}=0.2, \\
& \sigma_{\mathrm{LJ}}=3.30 \AA, \\
& E_{\mathrm{LJ}}=30.0 \mathrm{~K} .
\end{aligned}
$$

With the bend angles at their equilibrium values of $109^{\circ}$, the potential $V_{\text {dih }}$ has the following characteristics: transgauche barrier, $1770 \mathrm{~K}$; gauche energy, $360 \mathrm{~K}$; cis energy, $3180 \mathrm{~K}$.

The effect of the bend angles on the potential $V_{\text {dih }}$ is shown in Fig. 2(b). The number on each curve is the magnitude of both bend angles in the group of four pseudoatoms defining the torsion. We see that the transgauche barrier is almost independent of the bend angle in contrast to the position and energy of the gauche states.
When the bend angles exceed their equilibrium value of $109^{\circ}$ the position of the gauche state moves toward that of the cis conformation or its energy is lowered. A smaller bend angle has the opposite effect. The dependence of the cis energy and gauche-gauche barrier on the bend angle is more dramatic. One sees that for a bend angle of $112^{\circ}$ the gauche-gauche barrier can become even smaller than that for the trans-gauche transition. This introduces a gauchegauche transition mode not present in the usual representation of the dihedral torsion potential. ${ }^{27,28}$ Since the effect of the bend angles on $V_{\text {dih }}$ has not been investigated experimentally, it is impossible to conclude whether these results are quantitatively or just qualitatively correct.

\section{METHOD OF SIMULATION}

In the skeletal model of the alkanes, the nearestneighbor distances of the pseudoatoms are fixed. These constraints must be built into the integration scheme for the equations of motion, and two different approaches have been suggested previously. Both are based on a Cartesian coordinate representation and apply the method of Lagrange undetermined multipliers. In one scheme, ${ }^{18}$ the constraints are implemented directly with the distances being fixed at the preset values after each time step. The Lagrange multipliers which determine the constraint forces are found iteratively from a matrix equation. Since the number of iterations per time step may be quite large, this can greatly increase computation times particularly in long simulations.

The other approach ${ }^{31,32}$ is based on Gauss' principle of least constraint. It does not use the constraints directly but rather their second time derivatives. This has the advantage that the Lagrange multipliers are determined directly as the solution of a matrix equation and not iteratively. The disadvantage of this method is that the interatomic distances are no longer fixed but can drift due to numerical errors. Therefore, they must be checked regularly, and, if changed by more than a preset amount, reset to their original value.

We have chosen to use the latter approach. However, we have modified the original method of resetting the constrained distances ${ }^{32}$ which we found to be inefficient and unreliable. In our approach, we use the Gauss method only when all constrained distances are within a prescribed tolerance. When just one distance is found to be out of tolerance, the iterative method ${ }^{18}$ is used to determine the constraint forces necessary to fix all distances at their correct values at the next step. We have also implemented various other improvements in the Gauss approach as described in detail elsewhere. ${ }^{33}$

We have performed the integration of the equations of motion with a Gear predictor-corrector scheme. ${ }^{27,34}$ Since such schemes are not perfectly time reversible, there will be some drift in the total energy of the system in a microcanonical simulation. This drift is used to establish the time step to be used in the simulation. In the case of the butane monolayer, a time step of $0.002 \mathrm{ps}$ gave an energy drift of $0.03 \% / \mathrm{ps}$ at temperatures near the melting point $(150 \mathrm{~K})$ which was found to be acceptable. Since the hexane mono- 
TABLE III. Simulation parameters.

\begin{tabular}{lccrrc}
\hline \hline Monolayer & $x_{\text {box }}(\AA)$ & $y_{\text {box }}(\AA)$ & $a(\AA)$ & $b(\AA)$ & $\begin{array}{c}\text { No. of } \\
\text { molecules }\end{array}$ \\
\hline Butane & $63.96(=8 a)$ & $68.16(=8 b)$ & 7.995 & 8.52 & 128 \\
Hexane & $68.88(=13 a)$ & $68.16(=4 b)$ & 5.298 & 17.04 & 104 \\
\hline \hline
\end{tabular}

layer had a higher melting point $(225 \mathrm{~K})$, it was necessary to use a smaller time step of $0.001 \mathrm{ps}$ in order to achieve the same energy drift as for butane.

In constant-volume simulations of bulk phases, there are no restrictions on the simulation box size. However, this is not true when the system is a monolayer adsorbed on a substrate. In this case, the two-dimensional simulation box for the monolayer must be commensurate with the substrate lattice. This can be expressed as follows. Let the lattice vectors of the graphite basal plane be $a_{1}$ and $a_{2}$ with $a_{1}$ along the $x$ axis in Fig. 5. For a rectangular simulation box, the length of the side along the $x$ direction, $x_{\text {box }}$, is limited to the following values:

$$
x_{\text {box }}=a_{1} l_{x}=2.46 l_{x} \AA, \quad l_{x}=0,1,2,3,4, \ldots,
$$

and the side along the $y$ direction, $y_{\text {box }}$, to values

$$
y_{\text {box }}=\frac{\sqrt{3}}{2} a_{2} l_{y}=2.13 l_{y} \AA, \quad l_{y}=0,2,4,6,8, \ldots .
$$

To facilitate comparison of the simulation results with experiment, it is desirable to use box dimensions as large as the coherence length $L$ of films investigated by neutron diffraction. At low temperatures, $L$ values of $\sim 120 \AA$ have been inferred for the solid butane and hexane monolayers; ${ }^{3}$ these are presumably determined by the distance between defects on the surface of the graphite particles (e.g., between steps). However, in order to save computational time, it was not possible to use a cluster size as large as 120 $\AA$. A simulation box of size $\sim 68 \AA \times 68 \AA$ (see Table III for exact size) was chosen, giving a maximum cluster dimension of $\sim 90 \AA$. In Sec. V, we shall discuss possible effects on the melting transition associated with this small box size.

The simulations are done at a constant temperature corresponding to a canonical sampling. This is accomplished by adding a nonholonomic constraint on the kinetic energy ${ }^{32,35}$ calculated from the center-of-mass velocities. Since the intramolecular degrees of freedom are not constrained, the total kinetic energy and hence the instantaneous temperature fluctuates. However, the coupling between the intramolecular and center-of-mass degrees of freedom enables an equilibrium condition to be reached in which the temperature calculated from the atomic velocities oscillates about the value to which the center-of-mass velocities have been constrained. This condition is used to verify that the kinetic energy has been distributed evenly among the various degrees of freedom. Similarly, the potential energy associated with the bend and dihedral torsion angles is initially zero and is later examined to ensure that an equilibrium condition has been reached in which these energies oscillate about a constant value as a function of time.

At the beginning of a simulation run, the molecular configurations are those of the solid, orientationally ordered, herringbone structure obtained by minimizing the potential energy of the monolayer cluster at zero temperature (see Sec. II and Table II). As discussed in Sec. V, the lattice constants of the butane and hexane clusters are altered slightly from their calculated low-temperature values in Table II in order for corresponding molecules at the cluster edges to be at equivalent sites on the graphite substrate and hence satisfy the periodic boundary conditions. The initial atomic velocities are chosen from a Maxwell distribution at the given temperature. The duration of a run varied with temperature. Typically, the systems were allowed to run for $150-200$ ps while up to $200-300$ ps was required near transition temperatures for proper identification of the phases. All simulations were performed on the Cray Y-MP/832 at the Pittsburgh Supercomputing Center.

\section{MONITORING OF THE SIMULATION}

The simulation results are summarized by a set of thermodynamic functions and structural data. These results are computed as an average over samples within a time block of 10 ps with sampling every $0.1-0.2$ ps. After an initial thermalization run, data is accumulated over several time blocks. The variance in the data can be estimated by assuming the time blocks are statistically independent.

Various thermodynamic functions are recorded during a simulation including the intermolecular potential energy which we will show for both monolayers in the next section. The structural data obtained from the simulations may be divided into three groups as follows:

(1) Translational order

(a) atomic-pair distribution function;

(b) center-of-mass distribution function;

(c) structure factor corresponding to a low-energy electron diffraction pattern as discussed below;

(d) spherically averaged structure factor (defined below) for comparison with observed neutron diffraction patterns.

(2) Orientational order

(a) polar angle $\theta$ of the molecule's "long" axis (defined below);

(b) rolling angle $\psi$ around the long axis;

(c) azimuthal angle $\phi$ of the long axis.

(3) Molecular structure

(a) bend angles;

(b) dihedral torsion angles.

The instantaneous structure of the monolayer cluster is recorded at the end of each time block by projecting the atomic coordinates on the surface $(x y)$ plane. To characterize the translational order within the monolayer cluster quantitatively, it is of interest to calculate both the atomicpair and center-of-mass correlation functions which are calculated at periodic intervals during a time block and 


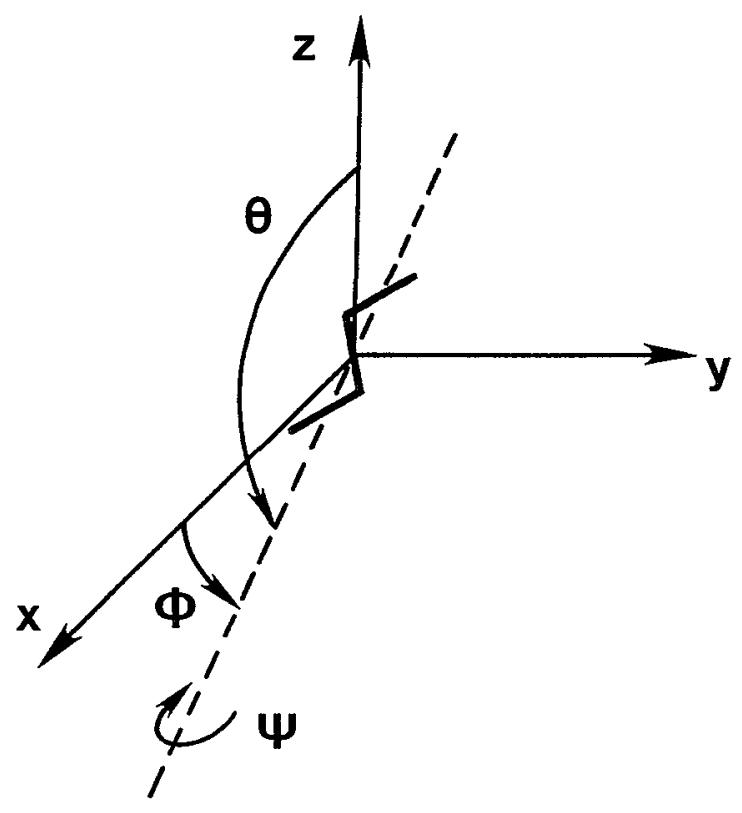

FIG. 3. Definition of the three angles specifying the molecular orientation in a coordinate system fixed to the graphite substrate. The $z$ axis is perpendicular to the graphite basal plane and the direction of the $x$ and $y$ axes is shown in Fig. 5. The "long" axis of the molecule (dashed line) is defined as the principal axis of the molecule with the smallest moment of inertia. Its direction is specified by the polar angle $\theta$ and azimuthal angle $\phi$. The rolling angle $\psi$ about the long axis is defined as the angle between the principal axis with the largest moment of inertia and the $x y$ plane. For a molecule in the trans configuration as shown, the principal axis with the largest moment of inertia is perpendicular to the plane of the pseudoatoms so that $\psi=90^{\circ}$ when this plane is parallel to the surface.

then averaged. It is important to note that the distribution function calculations are performed without imposing periodic boundary conditions which introduce false peaks. This has the drawback of introducing small errors in some of the peak intensities due to size effects.

In addition to these distribution functions, it is useful to calculate various structure factors such as the $2 \mathrm{D}$ Fourier transform of the real-space configuration:

$$
I_{\mathrm{LEED}} \propto \sum_{i>j} \cos \left[\mathbf{Q} \cdot\left(\mathbf{r}_{j}-\mathbf{r}_{i}\right)\right],
$$

where $\mathbf{Q}$ is a 2D wave vector in the $x y$ plane and $\mathbf{r}_{i}$ is the position vector of the $i$ th atom. In practice, the Fourier transform is calculated from an average structure obtained by sampling the atomic positions periodically during a time block. These Fourier transforms are similar to low-energy electron diffraction (LEED) patterns, and, for convenience, we shall refer to them as such. Although LEED experiments investigating the melting of butane and hexane monolayers on single-crystal graphite have not yet been performed, the calculated LEED patterns are still quite useful. They provide a sensitive means of characterizing structural properties of the monolayer such as thermal expansion and rotation of the film lattice vectors with respect to the graphite substrate.

It is also useful to calculate the spherically averaged structure factor $S(Q)$ given by the relation
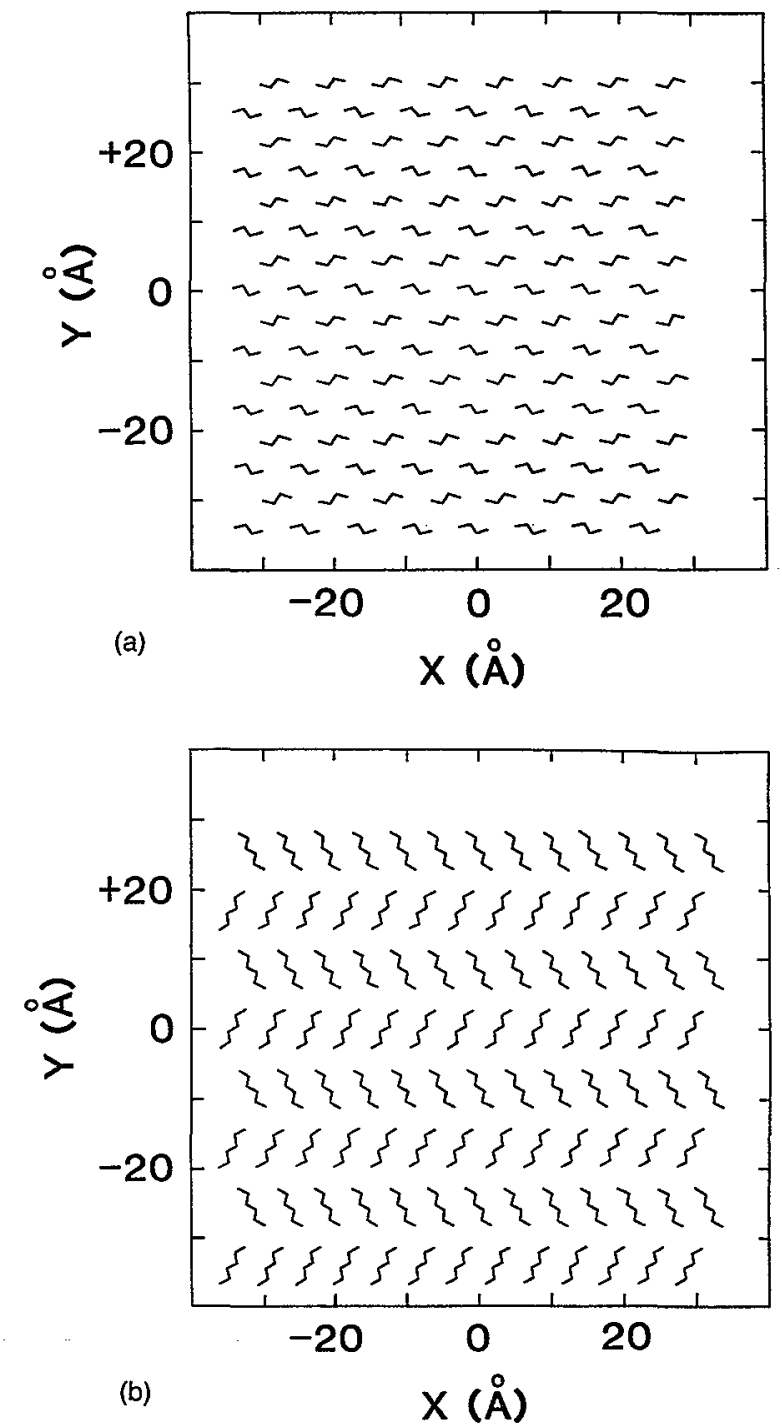

FIG. 4. The herringbone structure of the monolayers used as the initial configuration in the simulations: (a) butane and (b) hexane.

$$
I_{\mathrm{sph}} \propto \sum_{i, j} \frac{\sin \left(Q\left|\mathbf{r}_{j}-\mathbf{r}_{i}\right|\right)}{Q\left|\mathbf{r}_{j}-\mathbf{r}_{i}\right|} .
$$

The calculated $S(Q)$ can be compared with the observed neutron diffraction patterns from butane and hexane monolayers on powdered graphite substrates. ${ }^{17}$

Due to the great amount of computation time required, the structure factors are only evaluated for a few time blocks near the end of the simulation. It should be emphasized that, since the pair distribution functions are determined without periodic boundary conditions, finite size effects are present in the structure factors calculated from them.

To distinguish between orientationally ordered and disordered phases, three angles specifying the molecular orientation are of interest as defined in Fig. 3: the polar angle $\theta$ between a molecule's long axis and the $z$ direction perpendicular to the surface, the rolling angle $\psi$ about the molecule's long axis, and the in-plane azimuthal angle $\phi$ giving the direction of the molecule's long axis in the $x y$ 
plane. The "long" axis ( $\psi$ axis) is defined to be the principal axis of the molecule with the smallest moment of inertia. When the molecule is in the trans conformation, the principal axis with the largest moment of inertia is

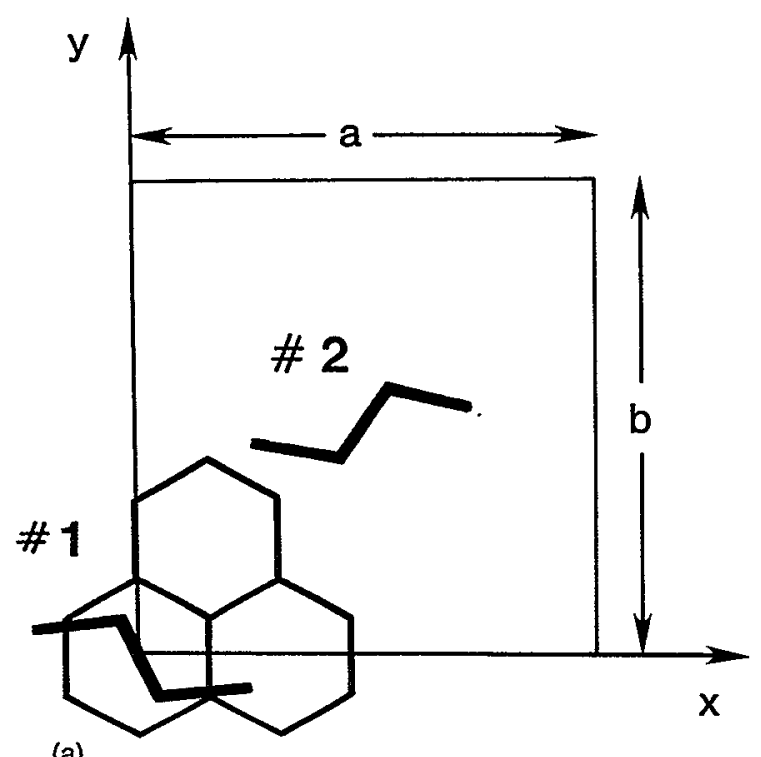

(a)

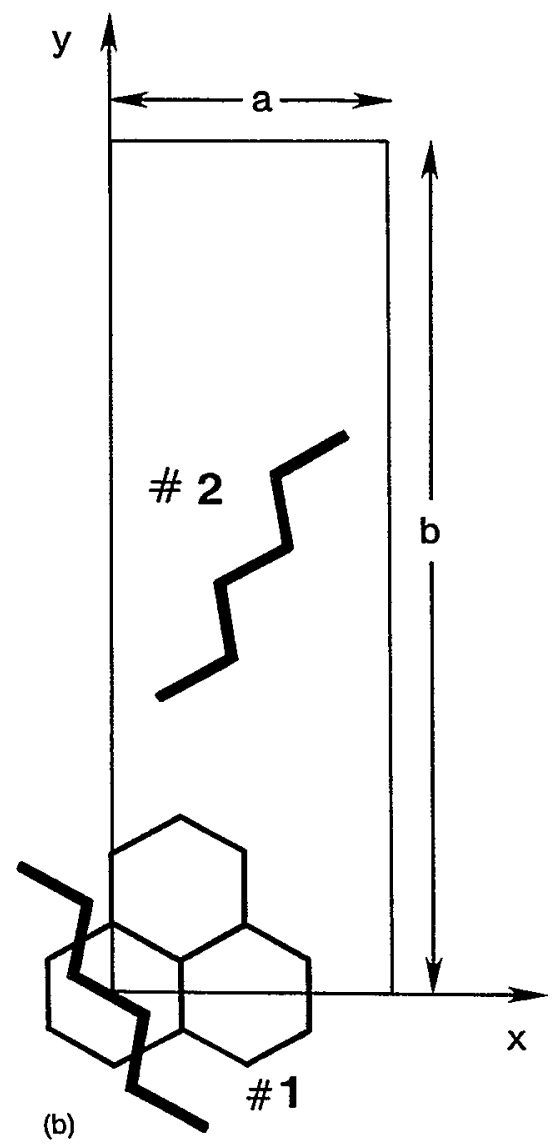

FIG. 5. Schematic diagram of the monolayer unit cells in the initial configuration of Fig. 4: (a) butane and (b) hexane. Some carbon hexagons of the graphite basal plane are included to show the relative orientation and size of the monolayer and graphite lattices. They do not indicate the correct adsorption site of minimum energy for molecule 1 . In both (a) and (b), molecule 2 is at the center of the cell. The $x$ and $y$ axes are in the graphite [110] and [100] directions, respectively. perpendicular to the plane of the pseudoatoms. The rolling angle $\psi$ is the angle between this axis and the surface plane.

Changes in the molecular structure are monitored with the bend angle and dihedral-torsion angle distribution functions. The latter is of particular interest, since it shows the distribution between trans and gauche conformations of the molecule.

\section{RESULTS}

The simulations produced a tremendous wealth of data which cannot all be presented here. In this section, we summarize the essential results, beginning with features common to both systems. The initial configurations of the molecules in the two monolayers are shown in Fig. 4. As described in Sec. II, these were found by minimizing the potential energy of monolayer clusters and yield the structural parameters listed in Table II. Upon heating these clusters to within $50 \mathrm{~K}$ of the melting point, some molecules were observed to rotate $180^{\circ}$ about their long axis. Further heating increases this flipping rate, but a uniform distribution in the $\psi$ angle of the molecules is never reached even at the highest temperatures of the simulations, 35 and $50 \mathrm{~K}$ above the melting point for butane and hexane monolayers, respectively. At these temperatures, most of the molecules librate with amplitudes up to $30^{\circ}$. That is, for molecules in the trans conformation (or nearly so), the plane containing the pseudoatoms tilts up to $30^{\circ}$
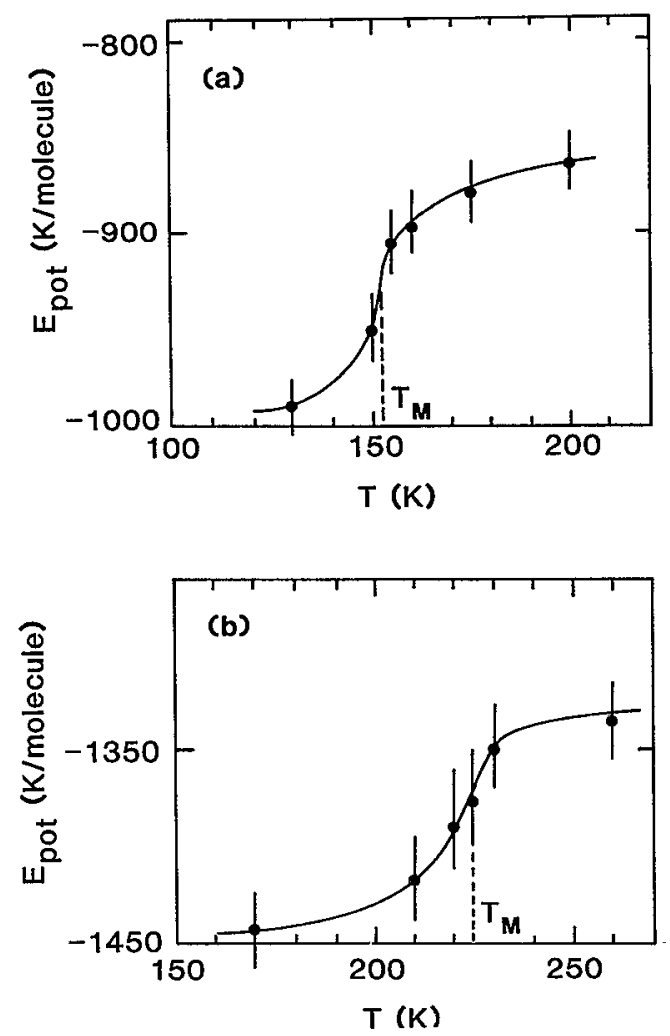

FIG. 6. The intermolecular potential energy of the monolayers as a function of temperature. The melting point is indicated as $T_{m}$ : (a) butane, $T_{m}=152 \mathrm{~K}$; (b) hexane, $T_{m}=222 \mathrm{~K}$. The potential energy functions are expressed in units of degrees Kelvin per molecule. 


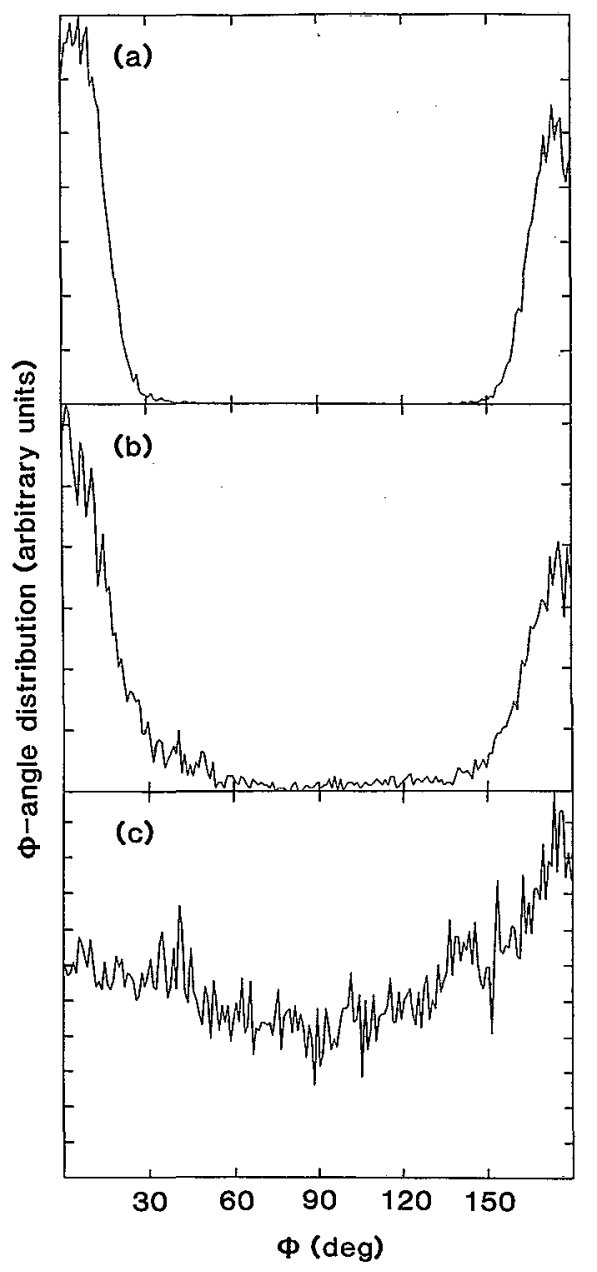

FIG. 7. Probability distribution of the azimuthal angle $\phi$ for the butane monolayer at several temperatures: (a) at $130 \mathrm{~K}$, well below the melting point estimated from the simulations; (b) at $150 \mathrm{~K}$, just below the estimated melting point; and (c) at $155 \mathrm{~K}$, just above the melting point. The two peaks in both (a) and (b) correspond to the $\phi$ values of the two sublattices of the low-temperature herringbone structure. At the highest temperature (c), angles between these two peaks begin to be populated as orientational order in the azimuthal angle is lost.

away from the surface. This fipping and concomitant librational motion has the effect of introducing a DebyeWaller factor in the calculated structure factors just as does the thermal motion in the internal molecular degrees of freedom. Thus it is difficult to separate the effects of librational and intramolecular motion in the calculated structure factors and the observed neutron diffraction patterns.

Another feature common to the simulations of both monolayers is that the molecules never stand on end with their long axis perpendicular to the surface, since this orientation is energetically very unfavorable. Instead, the simulations show an increasing number of molecules in the gauche conformation (more so for hexane) as the temperature is raised above the melting point. In the case of hexane, gauche molecules actually appear below the melting point. The importance of this feature for the melting process is discussed below.

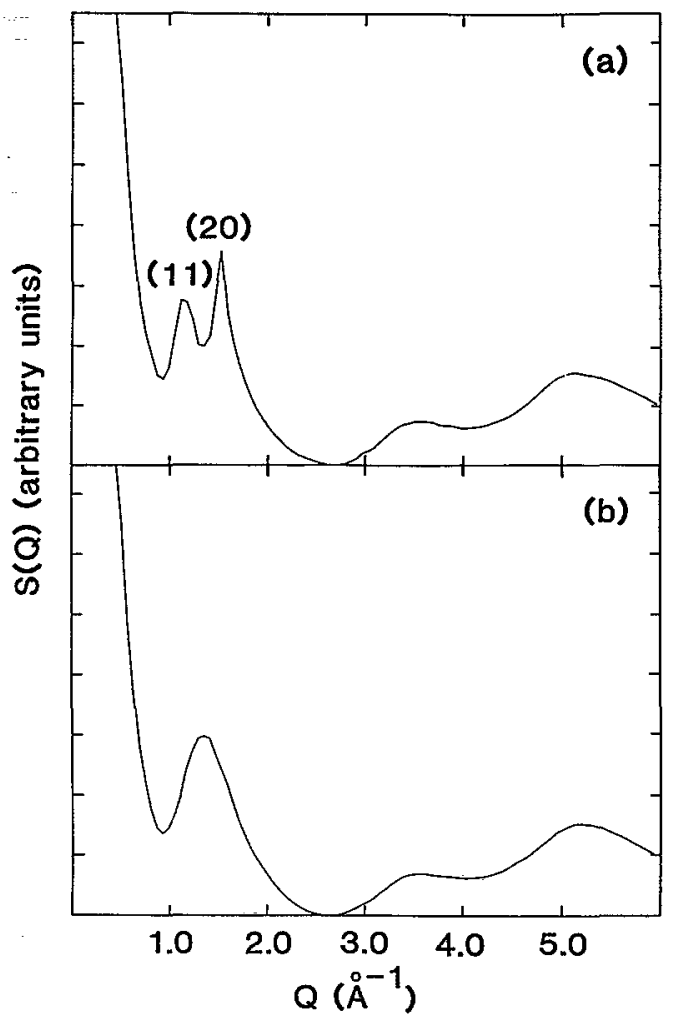

FIG. 8. Spherically averaged structure factor of the butane monolayer at two different temperatures. (a)At $T=150 \mathrm{~K}$, the Bragg peaks in the range 1-2 $\AA^{-1}$ show that the monolayer is crystalline with long-range translational order. (b) At $T=155 \mathrm{~K}$, just above the melting point, the broad peak at $\sim 1.5 \AA^{-1}$ is characteristic of a fluid phase.

For both monolayers, the melting temperature inferred from the simulations is $25 \%-30 \%$ higher than observed. This discrepancy probably results from a defect in the skeletal model used to represent the intermolecular interactions. It might also be caused by using too large of a corrugation amplitude in the molecule-substrate potential. However, it is unlikely that the skeletal model is overestimating the corrugation amplitude, since, as we have seen in Sec. II, the calculated zero-temperature monolayer structure for hexane is incommensurate with the graphite contrary to experiment. It is also possible that superheating or size effects could delay the melting point upon heating. Simulations are in progress to test the dependence of the melting point on sample size. Preliminary results on a system of 200 butane molecules (50\% larger than used here) show no change in the melting temperature. While the reason for the calculated melting point being higher than observed remains uncertain, its origin is probably the same for the two monolayers and therefore irrelevant for understanding the qualitative difference in their melting behavior.

\section{A. Butane}

Since the calculated film lattice vector along the $x$ axis in Fig. 5 is incommensurate with the graphite substrate ( $a=7.88 \AA$ in Table II), we are restricted in the choice of 
TABLE IV. Full width at half maximum (FWHM) in degrees of orientational angle distributions in the butane monolayer at various temperatures. Double entries for the azimuthal angle $\phi$ refer to the two different sublattices of the herringbone structure.

\begin{tabular}{lcccc}
\hline Temperature & & & & \\
Angle & $50 \mathrm{~K}$ & $80 \mathrm{~K}$ & $100 \mathrm{~K}$ & $120 \mathrm{~K}$ \\
\hline Polar angle, $\theta$ & 5.1 & 6.2 & 7.4 & 8.1 \\
Azimuthal angle, $\phi$ & $9.3,9.3$ & $11.1,11.1$ & $12.9,12.9$ & $20.0,20.0$ \\
Rolling angle, $\psi$ & 15.4 & 19.8 & 22.2 & 25.0 \\
\hline \hline
\end{tabular}

the simulation box size as discussed in Sec. III. For a system with eight film lattice vectors along both the $x$ and $y$ directions of the substrate, the simulation box dimensions would be

$$
\begin{array}{lll}
x_{\text {box }}=7.88 \times 8 \AA=63.01 \AA=2.46 & l_{x} \AA, & l_{x}=25.63 ; \\
y_{\text {box }}=8.55 \times 8 \AA=68.40 \AA=2.13 & l_{y} \AA, & l_{y}=32.10 .
\end{array}
$$

Since Eqs. (10) require integer values of $l_{x}$ and $l_{y}$, we have set $l_{x}=26$ and $l_{y}=32$. This results in a simulation box of dimensions $x_{\text {box }}=63.96 \AA$ and $y_{\text {box }}=68.16 \AA$ so that the film with lattice constants are $a=7.995 \AA$ and $b=8.52 \AA$

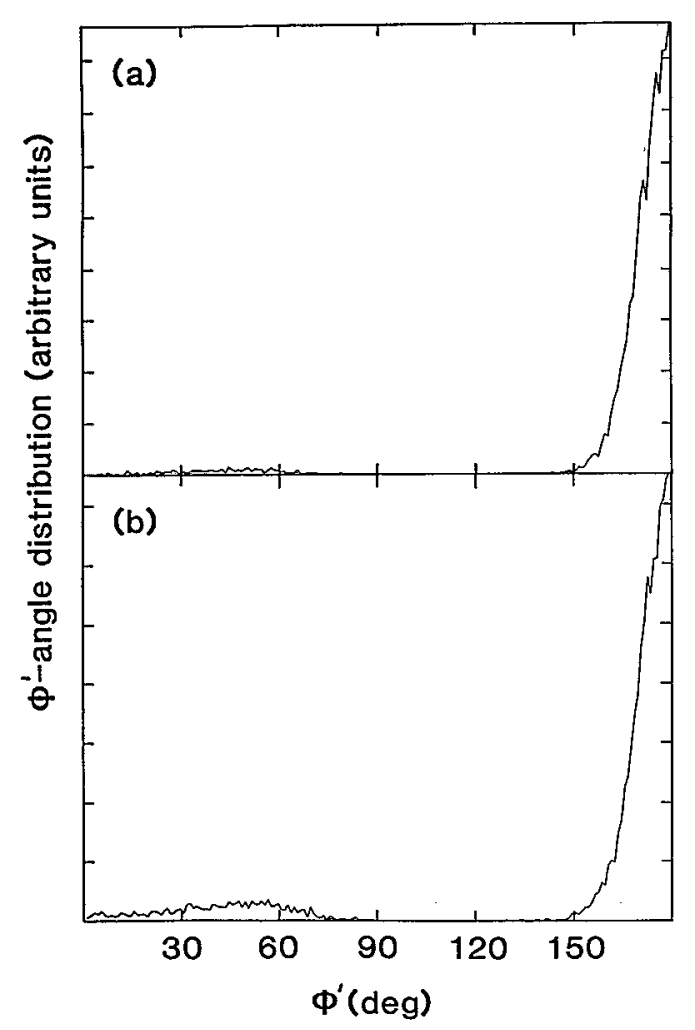

FIG. 9. The dihedral-torsion angle $\phi^{\prime}$ probability distribution of the hexane monolayer at two temperatures: (a) at $T=210 \mathrm{~K}$, just below the melting point; (b) at $T=225 \mathrm{~K}$, just above the melting point. Note that gauche molecules are present at both temperatures as can be seen from the finite value of the distributions near $\phi^{\prime}=60^{\circ}$. The distribution is calculated from the total number of gauche dihedral bonds. Since, in most cases, there is only one gauche bond per molecule, the fraction of gauche molecules can be approximated well by multiplying the fraction of gauche bonds obtained from this figure by a factor of 3 . along the $x$ and $y$ directions, respectively. Thus the butane simulations have been performed on a film which is initially expanded $\sim 1.5 \%$ in the $x$ direction and compressed $\sim 0.4 \%$ in the $y$ direction relative to the calculated zerotemperature monolayer structure. The simulation parameters for the butane monolayer are summarized in Table III and the relative orientation of the monolayer and graphite lattices is indicated in Fig. 5(a).

The butane monolayer simulations were performed at temperatures of $80,100,120,130,150,155,160,175$, and $200 \mathrm{~K}$. In Fig. 6(a), the intermolecular energy $E_{\text {pot }}$ is plotted as a function of temperature. A jump in the energy between 150 and $155 \mathrm{~K}$ indicates that a transition is occurring in this temperature range.

Great care was taken to establish the equilibrium phase at these temperatures. At $150 \mathrm{~K}$, the system still has the herringbone structure, although some defects have appeared. This is seen, for example, in the $\phi$-angle distributions (rotation angle of a molecule about the surface normal). At $130 \mathrm{~K}$ as shown in Fig. 7(a), two well-separated peaks are observed characteristic of the two different sublattices in the low-temperature herringbone phase. These two peaks still dominate the distribution at $150 \mathrm{~K}$ [Fig. 7 (b)], although there is some filling of the gap between them. The simulations were continued at this temperature for $250 \mathrm{ps}$ in order to establish the phase with more certainty. During this time, it was found that the level of the distribution between the peaks approached a stable value with small oscillations about it. A plot of the spherically averaged structure factor $S(Q)$ in Fig. $8($ a) is also consistent with this interpretation, since it clearly shows diffraction peaks characteristic of a herringbone phase with longrange translational order. Thus, we conclude that the phase at $150 \mathrm{~K}$ has a crystalline herringbone structure with a small number of defects.

A dramatic change in the $\phi$-angle distribution is observed at $155 \mathrm{~K}$ as shown in Fig. 7(c). The height of the distribution between the two peaks which are characteristic of the herringbone phase has risen markedly, and the two peaks have broadened indicating rotational disorder about the surface normal. The distributions of all three orientational angles are summarized in Table IV where the full width at half maximum (FWHM) of the peaks appearing in them are seen to increase as a function of temperature. At the same time, a loss in translational order has occurred by $155 \mathrm{~K}$ as can be seen in the plot of $S(Q)$ in Fig. 8(b). Here the two Bragg peaks at low $Q$ have been replaced by

TABLE V. Full width at half maximum (FWHM) in degrees of orientational angle distributions in the hexane monolayer at various temperatures. Double entries for the azimuthal angle $\phi$ refer to the two different sublattices of the herringbone structure.

\begin{tabular}{lllll}
\hline Temperature & & & & \\
Angle & $50 \mathrm{~K}$ & $100 \mathrm{~K}$ & $150 \mathrm{~K}$ & $170 \mathrm{~K}$ \\
\hline Polar angle, $\theta$ & 1.1 & 1.7 & 2.2 & 2.5 \\
Azimuthal angle, $\phi$ & $2.5,2.5$ & $3.9,3.9$ & $3.7,4.4$ & $5.0,5.1$ \\
Rolling angle, $\psi$ & 5.8 & 9.4 & 9.9 & 13.5 \\
\hline \hline
\end{tabular}


a single broad peak characteristic of a fluid phase. In this way, we conclude that a melting transition has occurred at $\sim 152 \mathrm{~K}$ characterized by a simultaneous loss of orientational and translational order.

It is of particular interest to compare the dihedraltorsion angle distributions in the butane monolayer at 150 and $155 \mathrm{~K}$ just below and above the melting point, respectively. One finds that none of the butane molecules are in a gauche conformation at $150 \mathrm{~K}$ and that only $1.5 \%$ of the molecules have transformed to the gauche state at $155 \mathrm{~K}$. This implies that the formation of gauche molecules plays no essential role in the melting process.

\section{B. Hexane}

As was the case for the butane monolayer, the hexane monolayer structure calculated at zero temperature is incommensurate with the graphite substrate in the direction of the $x$ axis in Fig. 5(b) but nearly commensurate in the $y$ direction. In Table II, we see that the calculated value at zero temperature of the lattice constant $a$ for the hexane monolayer is $5.37 \AA$ compared to the observed value of $4.92 \AA=2 a_{1}$ where $a_{1}=2.46 \AA$ is the graphite-basal-plane lattice constant. This results in a problem in the selection of the simulation box size similar to that encountered for butane. As listed in Table III, we have chosen a box size,

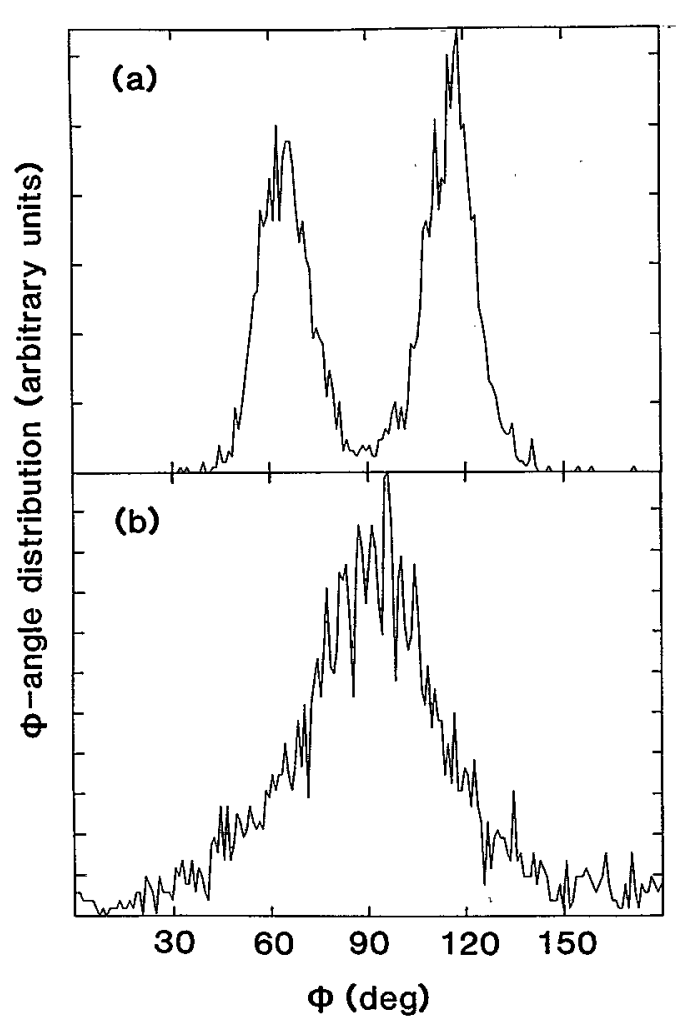

FIG. 10. Probability distribution of the azimuthal angle $\phi$ for the hexane monolayer at two temperatures: (a) At $210 \mathrm{~K}$, the two peaks correspond to $\phi$ values for the two sublattices of the low-temperature herringbone structure. (b) At $225 \mathrm{~K}$, only a single peak remains in the distribution characteristic of the RC phase. $x_{\text {box }}=68.88 \AA$ and $y_{\text {box }}=68.17 \AA$, which corresponds to a monolayer cluster of 104 hexane molecules of dimensions $13 a \times 4 b$ where $a=5.298 \AA$ and $b=17.04 \AA$. These lattice constants correspond to a compression from the zerotemperature calculated values of $1.3 \%$ and $0.1 \%$ along the $a$ and $b$ directions, respectively.

The hexane monolayer has been simulated at temperatures of $150,170,200,210,220,225,230$, and $250 \mathrm{~K}$. The plot of the intermolecular potential energy as a function of temperature in Fig. 6(b) indicates a transition in the temperature range of $220-225 \mathrm{~K}$.

The dihedral-angle distribution in Fig. 9(a) shows that below the melting point at $210 \mathrm{~K}$ about $9 \%$ of the hexane molecules are in the gauche conformation $\left(\phi^{\prime}=60^{\circ}\right)$, whereas no gauche molecules were observed for the butane monolayer below its melting point. The fraction of gauche hexane molecules increases to $\sim 30 \%$ just above the melting transition at $225 \mathrm{~K}$ [Fig. 9(b)]. Note also that there is a nonzero value of the distribution at $\phi^{\prime}=0$, the position of the gauche-gauche barrier [see Fig. 2(b)]. This is caused by the coupling of the bend and dihedral angles in the torsional potential.

The distributions of the orientational angles are summarized in Table $\mathrm{V}$ where the full width at half maxima (FWHM) is listed for various temperatures. We see that at each temperature the width of the polar angle $\theta$ distribution is less than that for butane. This narrower distribution results from a larger cost in potential energy to tilt the longer hexane molecule away from the surface. The temperature dependence of the angle distributions is seen to be the same for butane and hexane except for the azimuthal angle $\phi$. In Fig. 10(a), there is a double-peak distribution at $210 \mathrm{~K}$ characteristic of the herringbone structure. However, at $225 \mathrm{~K}$, just above the transition temperature, the double peak has been replaced by a single, broader peak. The origin of this single peak will be discussed below. For

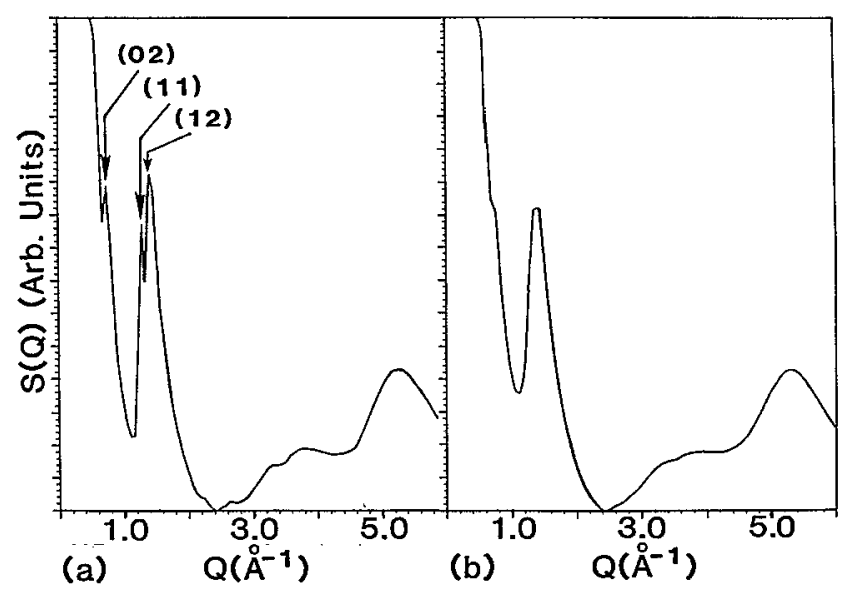

FIG. 11. Spherically averaged structure factor of the hexane monolayer at two different temperatures. (a) At $T=210 \mathrm{~K}$, the three Bragg peaks in the range $0.5-1.5 \AA^{-1}$ are consistent with a herringbone structure. (b) At $T=225 \mathrm{~K}$, the three Bragg peaks have been replaced by a single, broader peak which indicates some degree of translational order above the transition temperature. 


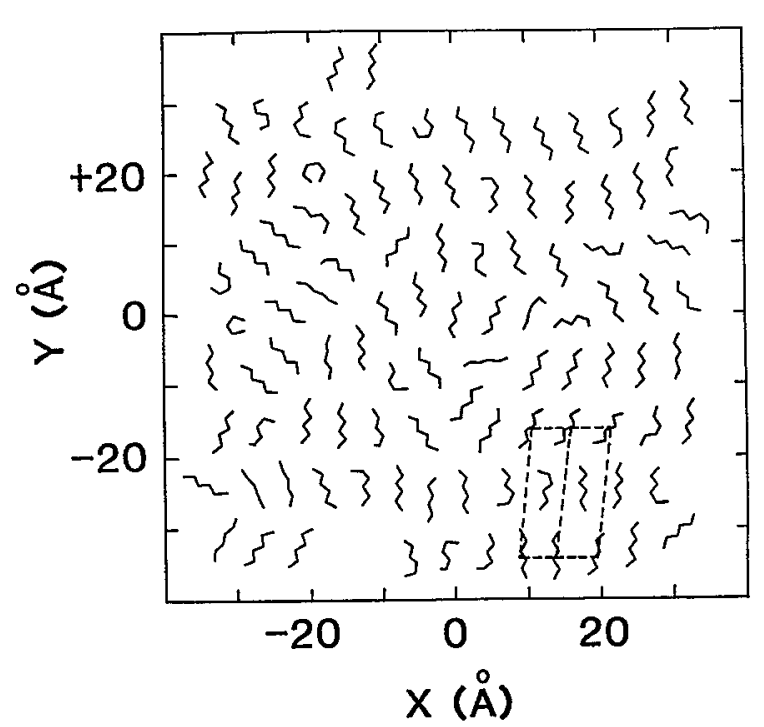

FIG. 12. Projection of the instantaneous molecular configurations onto the graphite surface from the hexane monolayer simulation at $T=225 \mathrm{~K}$ just above the melting transition.

now, we only note that its presence indicates a greater degree of azimuthal order just above the melting point than found for the butane monolayer [see Fig. 7(c)].

The spherically averaged structure factors $S(Q)$ for the hexane monolayer also show a dramatic change near $225 \mathrm{~K}$. Three diffraction peaks of the herringbone phase can be resolved at $210 \mathrm{~K}$ as labeled in Fig. 11(a). The large peak calculated in $S(Q)$ at $Q=0$ results from the finite size of the simulation box. At $225 \mathrm{~K}$, as shown in Fig. 11(b), the (02) peak has become a shoulder on the large peak at $Q=0$, while the (11) and (12) peaks have been replaced by a single peak. This peak is narrower than the one found for butane just above its melting point [Fig. 8(b)], indicating a greater degree of translational order in the hexane monolayer.

It is difficult to determine the structure of the hexane monolayer at high temperatures from just a single peak in $S(Q)$. Instead, it is helpful to study instantaneous configurations of the system. Such a "snapshot" is shown in Fig. 12 for the hexane system at $225 \mathrm{~K}$ just above the transition. As indicated by the dashed lines, one finds patches of solidlike material having a rectangular-centered (RC) structure in which the molecules are predominantly in their trans configuration. These patches are immersed in a fiuid or disordered phase containing a significant concentration of gauche molecules. As the simulation progresses, we observe that areas once occupied by the solidlike patches later become disordered (and vice versa). This behavior is characteristic of systems with coexisting phases.

The LEED pattern in Fig. 13 supports this interpretation of two-phase coexistence. The presence of rings of weak intensity together with diffraction spots is consistent with a system of coexisting fluid and crystalline phases. In order to index the spots in the LEED pattern, it is helpful to return to the "snapshot" of the system at the same

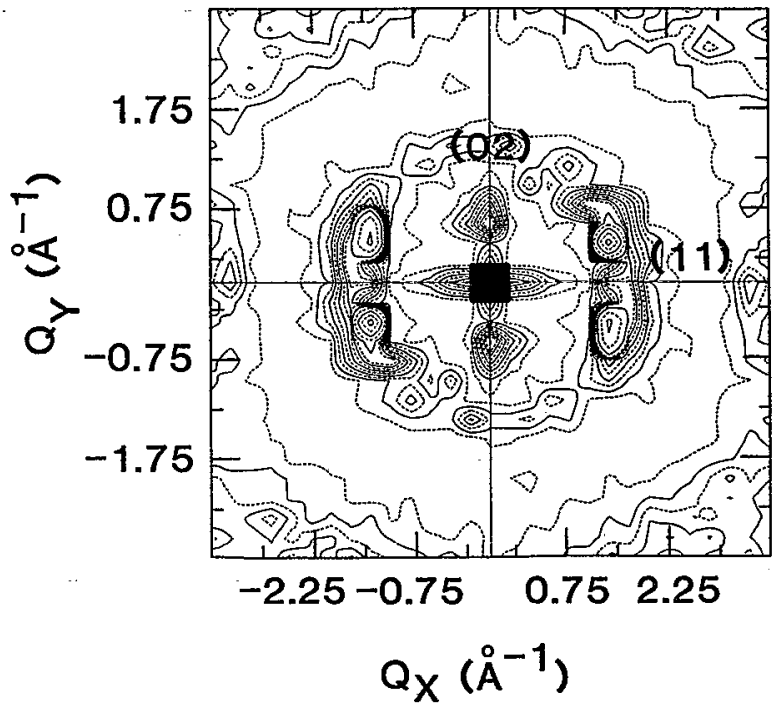

FIG. 13. Calculated LEED pattern for the hexane monolayer at $225 \mathrm{~K}$ just above the transition temperature. Intense spots like the one at the center appear to have a square shape due to imperfections in the contour plotting routine.

temperature of $225 \mathrm{~K}$ in Fig. 12. In the solidlike patches, one can identify rows of trans molecules lined up side by side with adjacent rows of molecules interlocked to form a rectangular-centered ( $R C$ ) structure. As illustrated schematically in Fig. 14, this structure consists of two identical sublattices while in the low-temperature herringbone phase these sublattices have different in-plane azimuthal orientations of the molecules. Thus the presence of an RC structure at high temperatures is consistent with the transition from a two-peak to a single-peak distribution of the $\phi$ angles seen in Fig. 10.

For an RC structure, Bragg spots in a LEED pattern which have an even-odd combination of the Miller indices are absent. This suggests indexing the spots in Fig. 13 as the (11) and (02), assuming approximately the same density for the herringbone and RC phases. The (11) spot in the LEED pattern would then correspond to the dominant
Low temperature

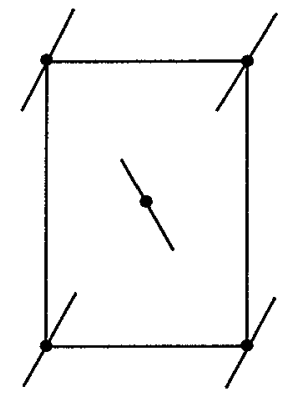

Herringbone structure
High temperature

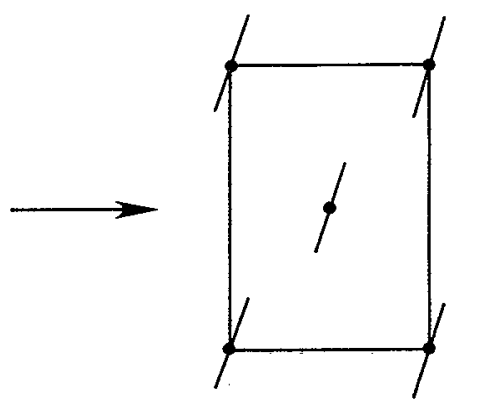

Rectangular-centered structure
FIG. 14. Comparison of the herringbone and rectangular-centered structures. 
peak at $Q=1.4 \AA^{-1}$ in the spherically averaged structure factor $S(Q)$ plotted in Fig. 11(b) and the (02) spot to the shoulder on the large $Q=0$ peak. These peak identifications also explain why the one at $Q=1.4 \AA^{-1}$ does not appear to broaden above the transition. At $225 \mathrm{~K}$ it results from only the (11) refiection of the RC phase, whereas at $210 \mathrm{~K}$ it consists of the overlapping (11) and (12) peaks of the herringbone phase. Thus the (11) peak of the RC phase actually has about twice the width of those of the low-temperature herringbone phase as one would expect from the RC patch size in Fig. 12.

Analysis of the high-temperature neutron diffraction patterns of a hexane monolayer adsorbed on an exfoliated graphite substrate is consistent with the presence of an RC structure and yields a characteristic patch dimension of $\sim 15 \AA .{ }^{3,5}$ However, these fits to the diffraction profile require the RC structure to be $10 \%-20 \%$ denser than the low-temperature herringbone phase. The simulations are consistent with this patch size (see Fig. 12) but do not allow a precise determination of the molecular density within a patch. Clearly, it would be desirable to perform molecular dynamics (MD) simulations on larger hexane clusters in an effort to obtain better values for the lattice constants of the RC phase. Also, LEED experiments would be helpful to corroborate the lattice constants inferred from the neutron diffraction experiments.

It is difficult to determine from the observed neutron diffraction patterns whether there is a fluid phase coexisting with the presumed $\mathrm{RC}$ patches at high temperatures. Such a fluid would contribute relatively weak and broad peaks to the diffraction pattern which are difficult to separate from the Bragg peaks of the solid component. Quasielastic neutron scattering experiments are now in progress $^{36}$ to compare the molecular diffusion rates in the butane and hexane monolayers. These may help to confirm that a fluid phase is coexisting with solidlike patches in the case of hexane.

In the disordered fluid phase, many molecules are observed to be in the gauche conformation (see Fig. 12). We suggest that the formation of gauche molecules may, in fact, be a prerequisite for melting to occur. The hexane molecules in the trans conformation could be too long to allow development of rotational disorder about the surface normal. The molecules can reduce their footprint on the surface by transforming to the gauche state. This creates space for neighboring trans molecules to disorder orientationally in the plane. In Refs. 16 and 17, we provide further evidence for this mechanism of melting and discuss how reduction of the molecular footprint is achieved differently in the case of the butane monolayer.

\section{DISCUSSION}

We can summarize the results of these simulations by saying that the melting process of the butane monolayer involves a simultaneous breakdown of the molecular orientational order and translational order characteristic of the herringbone structure. A 2D liquid-gas phase is formed upon melting in which none or only a very few of the molecules are in the gauche conformation.
The monolayer melting process of the longer hexane molecule differs from that of butane in that there remains a significant amount of translational order above the transition temperature and an appreciable number of molecules transform to the gauche conformation in the neighborhood of the transition. We have interpreted its structure above the transition as small patches of monolayer solid having a rectangular-centered structure in coexistence with a 2D fluid phase having a large fraction of gauche molecules.

Our tentative conclusion from these studies is that molecular flexibility plays an important role in the melting of the hexane monolayer but not for butane. We have suggested that, in their trans conformation, the hexane molecules are too long for rotational disorder about the surface normal to develop. By transforming to the gauche state, the molecules can reduce their footprint and thus create space for trans molecules to disorder orientationally about the surface normal. This hypothesis that the melting of the hexane monolayer is initiated by the trans-gauche transformations of some of the molecules can be tested by using more rigid molecules in the simulations. We have shown in Ref. 16 that, if the trans-gauche barrier in the dihedraltorsion potential is tripled, the melting point of the hexane monolayer increases by $\sim 75 \mathrm{~K}$. This supports the view that gauche formation is initiating the melting process. The simulations indicate that melting of the rigid-molecule monolayer eventually occurs when there is sufficient thermal energy for the molecules to tilt away from the surface (an increase in the polar angle $\theta$ ). Thus tilting provides an alternative mechanism for the molecules to reduce their footprint on the graphite surface.

For the shorter butane molecule, the tilting mechanism for footprint reduction appears to be more favorable energetically than a conformational change. Tripling of the trans-gauche barrier for the butane molecules did not change the monolayer melting point. ${ }^{16}$ Furthermore, if the magnitude of the molecule-substrate binding energy is tripled thereby inhibiting tilting, the butane monolayer melting point increases by $\sim 35 \mathrm{~K}$.

An important feature of the present study is that the same intermolecular and molecule-substrate interactions have been used for both the butane and hexane monolayer simulations. The only parameter which has changed is the length of the molecule. Therefore, we can have some confidence that the qualitatively different melting behavior of the monolayers results from the different molecular aspect ratio. We note, though, that at a quantitative level there may be defects in the potentials used to represent the interactions. For both monolayers, the melting point inferred from the simulations is about $25 \%$ higher than observed experimentally. Future work is planned to investigate improvements in the potentials particularly in the representation of the corrugation in the molecule-substrate holding potential. We also intend to study the effect of cluster size on the melting transition and the structure of the hightemperature phases of both monolayers.

The main purpose of this paper has been to describe in detail the methods used to perform molecular dynamics simulations over a wide range of temperature on monolay- 
ers of short alkane molecules adsorbed on a graphite substrate. Obviously, the ultimate value of these simulations lies in their ability to interpret previous experiments on these systems and suggest new ones. A detailed comparison between the simulations and experiments will be given in a future publication. ${ }^{5}$ In general, there is qualitative consistency between the simulated and observed film structures over the entire temperature range investigated. A possible discrepancy between the simulations and the neutron diffraction experiments involves the high-temperature rectangular-centered phase of the hexane monolayer. Analysis of the neutron diffraction profile yields a density of this phase which is $10 \%-20 \%$ larger than that of the lowtemperature herringbone structure. Simulations on larger hexane clusters are required in order to test this finding. Also, it is difficult to confirm from the neutron diffraction patterns the presence of a coexisting fluid phase at high temperatures in the hexane monolayer. Quasielastic neutron scattering experiments now being conducted ${ }^{36}$ should be able to observe diffusional motion characteristic of a fluid phase in the hexane monolayer as well as compare diffusion rates with those in the butane monolayer.

\section{ACKNOWLEDGMENTS}

This work was supported by The Danish Natural Science Research Council Grant No. M 11-7015, the U.S. NSF Grants No. DMR-8704938 and No. DMR-9011069, and the Pittsburgh Supercomputing Center Grant No. DMR-880008P.

${ }^{1} \mathrm{H}$. Taub, in The Time Domain in Surface and Structural Dynamics, Vol. 228 in NATO Advanced Study Institute, Ser. C, edited by G. J. Long and F. Grandjean (Kluwer, Dordrecht, 1988), p. 467.

${ }^{2} \mathrm{~K}$. W. Herwig, J. C. Newton, and H. Taub (unpublished).

${ }^{3}$ J. C. Newton, Ph.D. thesis, University of Missouri-Columbia, 1989 (unpublished).

${ }^{4}$ J. Krim, J. Suzanne, H. Shechter, R. Wang, and H. Taub, Surf. Sci. 162, 446 (1985).

${ }^{5}$ J. C. Newton, J. R. Dennison, S.-K. Wang, H. Taub, F. Y. Hansen, J. Suzanne, and $H$. Shechter (unpublished).

${ }^{6}$ G. J. Trott, H. Taub, F. Y. Hansen, and H. R. Danner, Chem. Phys. Lett. 78, 504 (1981).

${ }^{7}$ G. J. Trott, Ph.D. thesis, University of Missouri-Columbia, 1981 (unpublished).

${ }^{8}$ J. P. Coulomb, M. Bienfait, and P. Thorel, J. Phys. (Paris) 42, 293 (1981).
${ }^{9}$ H. K. Kim, Q. M. Zhang, and M. H. W. Chan, Phys. Rev. Lett. 56, 1579 (1986).

${ }^{10}$ J. Z. Larese, L. Passell, A. D. Heidemann, D. Richter, and J. P. Wicksted, Phys. Rev. Lett. 61, 432 (1988).

${ }^{11}$ H. Taub, G. J. Trott, F. Y. Hansen, H. R. Danner, J. P. Coulomb, J. P. Biberian, J. Suzanne, and A. Thomy, in Ordering in Two Dimensions, edited by S. K. Sinha (North-Holland, New York, 1980), p. 91.

${ }^{12}$ J. M. Gay, J. Suzanne, and R. Wang, J. Phys. (Paris) Lett. 46, L425 (1985).

${ }^{13}$ J. P. Coulomb and M. Bienfait, J. Phys. (Paris) 47, 89 (1986).

${ }^{14}$ M. A. Moller and M. L. Klein, J. Chem. Phys. 90, 1960 (1989).

${ }^{15}$ S. Leggetter and D. J. Tildesley, Ber. Bunsenges. Phys. Chem. 94, 285 (1990).

${ }^{16}$ F. Y. Hansen and H. Taub, in Phase Transitions in Surface Films 2, Vol. 267 in NATO Advanced Study Institute, Ser. B edited by H. Taub, G. Torzo, H. J. Lauter, and S. C. Fain, Jr. (Plenum, New York, 1991), p. 153.

${ }^{17}$ F. Y. Hansen and H. Taub, Phys. Rev. Lett. 69, 652 (1992).

${ }^{18}$ J. P. Ryckaert, G. Cicotti, and H. J. C. Berendsen, J. Comput. Phys. 23, 327 (1977).

${ }^{19}$ T. A. Weber, J. Chem. Phys. 69, 2347 (1978).

${ }^{20}$ T. A. Weber, J. Chem. Phys. 70, 4277 (1979).

${ }^{21}$ D. A. McQuarrie, Statistical Mechanics (Harper and Row, New York, 1976).

${ }^{22}$ M. Pear and J. H. Weiner, J. Chem. Phys. 71, 212 (1979).

${ }^{23}$ J. P. Ryckaert and A. Bellemanns, Discuss. Faraday. Soc. 66, 95 (1978).

${ }^{24}$ W. A. Steele, The Interaction of Gases with Solid Surfaces (Pergamon, Oxford, 1974).

${ }^{25}$ M. Abramowitz and I. A. Stegun, Handbook of Mathematical Functions (Dover, New York, 1970).

${ }^{26}$ Note that the hexane monolayer is observed to be commensurate in the graphite [110] direction ( $x$ axis in Fig. 5) whereas the butane is not. Experiment and simulation agree that the long axis of the butane molecule is nearly parallel to the [110] direction, whereas that of hexane is more nearly perpendicular to it.

${ }^{27}$ W. F. van Gunsteren, H. J. C. Berendsen, and J. C. Bellemanns, Mol. Phys. 44, 69 (1981).

${ }^{28}$ J. A. Pople, J. S. Brinkley, and R. Seeger, Int. J. Quant. Chem. Symp. 10, 1 (1976).

${ }^{29}$ H. D. Stidham and J. R. Durig, Spectrochim. Acta 42A, 105 (1986).

${ }^{30}$ K. Raghavachari, J. Chem. Phys. 81, 1383 (1984).

${ }^{31}$ D. J. Evans, W. G. Hoover, B. H. Failor, B. Moran, and A. J. C. Ladd, Phys. Rev. A 28, 1016 (1983).

${ }^{32}$ R. Edberg, D. J. Evans, and G. P. Morriss, J. Chem. Phys. 84, 6933 (1986).

${ }^{33}$ F. Y. Hansen (unpublished).

${ }^{34} \mathrm{C}$. W. Gear, Numerical Initial Value Problems in Ordinary Differential Equations (Prentice-Hall, New York, 1971).

${ }^{35}$ S. Nose, J. Chem. Phys. 81, 511 (1984).

${ }^{36} \mathrm{~K}$. W. Herwig, P. Dai, Z. Wu, H. Taub, T. O. Brun, F. Trouw, and F. $\mathrm{Y}$. Hansen (unpublished). 